ZhangFei Su, J. Jay Leitch, Fatemeh Abbasi, Robert J. Faragher, Adrian L. Schwan, Jacek

Lipkowski, "EIS and PM-IRRAS Studies of alamethicin ion channels in a tethered lipid bilayer",J.Electroanal.Chem. 2018,812,213-220

\title{
EIS and PM-IRRAS Studies of alamethicin ion channels in a tethered lipid bilayer
}

\author{
ZhangFei Su, J. Jay Leitch, Fatemeh Abbasi, Robert J. Faragher, Adrian L. Schwan, \\ Jacek Lipkowski* \\ Department of Chemistry, University of Guelph, Guelph, Ontario N1G 2W1, Canada \\ E-mail: jlipkows@uoguelph.ca
}

\begin{abstract}
Alamethicin was reconstituted into the DPhPC/DPTL tethered bilayer lipid membrane (tBLM) to gain a better understanding of ion pore formation. The properties of the ion-conductive alamethicin pores were investigated by electrochemical impedance spectroscopy (EIS) and polarization modulation infrared reflection absorption spectroscopy (PM-IRRAS) as a function of the peptide concentration within the membrane. At low peptide concentrations $(\leq 5 \%)$, alamethicin molecules adopt a surface state where the tilt angles of the helices are large with respect to surface normal. The highest membrane resistance is observed when alamethicin molecules adopts the surface state. At alamethicin concentrations ranging from 10 to $20 \mathrm{~mol} \%$, the tilt angles of the helices decrease to lower values signifying that the alamethicin molecules are inserting into the tBLM. Consequently, the membrane resistivity is also lowered indicating that the peptides are forming ion conductive channels. The results show an excellent correlation between molecular information concerning the orientation and conformation of alamethicin molecules obtained from PM-IRRAS measurement and electrochemical properties of the membrane measured by EIS. These findings reveal that alamethicin concentration within the film is directly related to the ion conductance
\end{abstract}


across membrane.

Dedicated to Professor Renata Bilewicz for her $65^{\text {th }}$ birthday and in recognition of her contributions to electrochemistry

Keywords: alamethicin, tethered bilayer lipid membrane, ion channels, EIS, PM-IRRAS

\section{Introduction}

A biological membrane acts as a barrier allowing selective communication between the interior and exterior of a biological cell. Natural cell membranes are highly complex and consist of numerous components, including phospholipids, cholesterol, proteins, oligosaccharides etc. Due to the complexity of the biological membrane, research in this field is frequently performed on simplified biomimetic models [1,2]. A tethered bilayer lipid membrane (tBLM) is an example of a typical biomimetic membrane where a self-assembled thiolipid molecule is anchored to a gold electrode surface [3]. Gold is currently one of the most popular substrates since it is easily functionalized by thiol-based lipids, and is amenable to membrane characterization by a wide range of surface-sensitive techniques. A popular tethered bilayer membrane, developed by Schiller et al., was created by first self-assembling a monolayer of 2,3-di-O-phytanyl-sn-glycerol-1-tetraethylene glycol-DL- $\alpha$-lipoic acid ester (DPTL) at a gold surface and then fusing unilamellar phospholipid vesicles to the surface of theself-assembled thiolipid monolayer [4]. The DPTL molecule consists of a lipoic acid head group that serves as an anchor to the gold surface. The lipoic acid is connected to two hydrophobic diphytanyl chains, which create inner (cytosolic) leaflet of the bilayer, by a tetraethylene glycol chain, which acts as a hydrophilic spacer separating the metal surface from hydrophobic diphytanyl chains. The outer (extracellular) leaflet is formed by fusion of phospholipid vesicles. In this design, the separation between the hydrophobic portion of the membrane and the gold electrode surface allows for the inclusion of an aqueous sublayer, which mimics the cytosol in biological cell 
membranes. In addition, tethered bilayers display long-term stability at gold surfaces making them attractive platforms for studying membrane-active proteins/peptides [5, 6] and developing new biosensors [7].

Alamethicin (Alm) is an antimicrobial peptide produced by the fungus Trichoderma viride [8]. Alamethicin contains 20 amino acid residues with a high proportion of 2-aminoisobutyric acid (Aib) residues, which induces a helical secondary structure. X-ray diffraction studies of alamethicin confirm that the predominantly helical secondary structure of the alamethicin peptide [9] is composed of an $\alpha$-helix subunit at the $\mathrm{N}$-terminus connected to a 310 -helical structure at the $\mathrm{C}$-terminus by a proline residue at position 14. This transition at the Pro14 residue forms a bend in the helical structure of the alamethicin molecule. The voltage-gated ion pore properties of alamethicin were recognized 50 years ago. [10] Due to its simple peptide structure, alamethicin has become a widely used model for studying the mechanistic behavior of voltage-gated ion pore formation and conductance [11]. Investigations of the alamethicin ion pore properties in a model membrane provide insight into the mechanisms of their antimicrobial activity and aid in the design and development of new antimicrobial drugs [12].

Recently, we have investigated the pore forming properties by incorporating alamethicin into a 1,2-di-O-phytanyl-sn-glycero-3-phosphocholine ( $\mathrm{DPhPC}$ ) bilayer supported at the gold electrode surface [13]. In our previous study, the membrane was directly deposited onto the metal surface. In the present work, various concentrations of alamethicin were reconstituted into DPhPC/DPTL bilayers to assess the impact of the metal on the insertion of a transmembrane peptide, such as alamethicin. The hydrophilic tetraethylene glycol chain of the DPTL molecule forms an architecture where the membrane is separated from the metal by an aqueous reservoir that provides adequate space and fluidity for transmembrane peptide insertion. Electrochemical impedance spectroscopy (EIS) was used to measure the effect of alamethicin on the membrane resistivity and polarization modulation infrared reflection absorption spectroscopy (PM-IRRAS) was employed to determine the orientation and conformation of alamethicin molecules in the tBLMs. The results of this paper offer 
new insight into the relationship between the concentration of alamethicin and membrane resistivity. In the past, several models have been proposed to describe pore formation by alamethicin molecules in a biomimetic membrane. These models assumed that the voltage driven pore formation involves a reorientation of peptide molecules from the membrane surface into the membrane interior, followed by peptide aggregation into a transmembrane pore $[11,14,15]$. Although these models provide an accurate description of the membrane conductivity data, they are heuristic in nature because the molecular orientations for the closed and open states of the pore are assumed, but not experimentally proven. The concentration dependent presence of alamethicin molecules at the membrane surface (surface state) and their insertion into the membrane was observed in X-ray diffraction studies of multiple bilayers [16-21] These two states also were observed in the sum frequency generation (SFG) studies of a bilayer supported at a surface of a $\mathrm{CaF}_{2}$ window [22, 23]. Although, the X-ray diffraction and the SFG findings provided important information about the concentration dependent interaction of alamethicin molecules with biomimetic membranes, these systems were not allowing measurement of the membrane conductivity. In this paper, a comparison of the spectroscopic and EIS data allows for correlations between the changes in the membrane conductivity with the changes in molecular orientation of the membrane constituents. These correlations provide unique molecular level information about the characteristics of the alamethicin in the opened and closed states of ion conductive pores.

\section{Experiment}

\subsection{Materials}

Alamethicin, from Trichoderma viride (Alm, 98\%), potassium perchlorate $\left(\mathrm{KClO}_{4}, 99 \%\right)$, and deuterium oxide $\left(\mathrm{D}_{2} \mathrm{O}, 99.9\right.$ atom \% D) were purchased from Sigma-Aldrich. 1,2-di-O-phytanyl-sn-glycero-3-phosphocholine (DPhPC) was obtained from Avanti Polar Lipids. All the chemicals were used as purchased without further purification. 2,3-di-O-phytanyl-sn-glycerol-1-tetraethylene glycol-DL- $\alpha$-lipoic 
acid ester (DPTL) was synthesized and purified at the University of Guelph [24]. All aqueous solutions were prepared using ultrapure water procured from a Milli-Q (EMD Millipore, Bedford, MA) water system (resistivity > $18.2 \mathrm{M} \Omega \mathrm{cm}$ ).

The single crystal gold (111) electrodes, which were grown, oriented, and cut in our laboratory [25], were used as the working electrodes in the electrochemical and PM-IRRAS experiments. The gold (111) electrode was flame-annealed and cooled in air prior to use. All potentials reported in this paper are quoted versus the $\mathrm{Ag} / \mathrm{AgCl}$ (saturated $\mathrm{KCl}$ ) electrode. All experiments were performed at room temperature (20 $\left.\pm 2^{\circ} \mathrm{C}\right)$.

\subsection{Assembly of the Alm:DPhPC( $x: 100-x) / D P T L$ tethered bilayer at the gold (111)} surface

Alm:DPhPC/DPTL bilayers with different peptide to lipid (P/L) ratios were assembled at the gold (111) surface via self-assembly of a DPTL monolayer by immersing the gold (111) electrode in a $0.1 \mathrm{mg} \mathrm{mL}^{-1}$ DPTL methanolic (HPLC grade, Fisher Scientific) solution for $12 \mathrm{~h}$. Afterwards, the electrode was rinsed with excess of

methanol and ultrapure water, and then transferred to $1 \mathrm{mg} \mathrm{mL}^{-1} \operatorname{Alm}: \operatorname{DPhPC}(x: 100-x)$ vesicle solutions with variable alamethicin concentrations $(x \mathrm{~mol} \%)$ for an additional $12 \mathrm{~h}$ to deposit the Alm:DPhPC outer leaflet on top of the DPTL monolayer. After completion of the bilayer by vesicle fusion, the tBLM covered electrodes were carefully rinsed with ultrapure water to remove all residual molecules. A schematic diagram of the Alm:DPhPC/DPTL bilayer is presented in Fig. 1(a).

The Alm:DPhPC vesicle solution was prepared according to Ref. [26]. Individual stock solutions of both alamethicin and DPhPC dissolved in chloroform $(99.8 \%$, Fisher Scientific) were added to test tubes to give the total peptide and lipid mass of $\sim 1 \mathrm{mg}$. By adjusting the volume ratio between the peptide and lipid stock solutions, different P:L ratios in the mixture can be achieved. The mixed solution was dried by vortexing in the test tube under a flow of argon creating a thin Alm:DPhPC coating on the inside wall of the test tube. Dried Alm:DPhPC films were placed in a vacuum desiccator before use to remove the residual solvent. Finally, $1 \mathrm{~mL}$ of Milli-Q water was added to the test tube 
and the mixture was sonicated for 30 minutes to obtain the Alm:DPhPC vesicle solution.

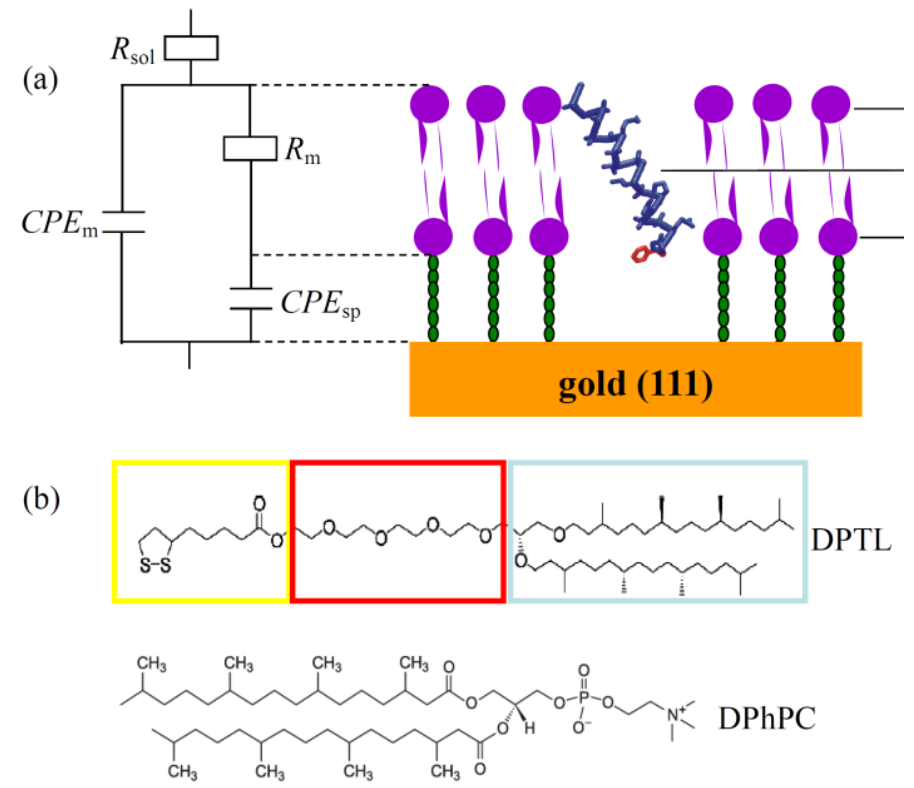

Fig. 1 (a) Schematic diagram of the $\operatorname{Alm}: \operatorname{DPhPC}(x: 100-x) / \mathrm{DPTL}$ bilayer at the gold (111) surface with the corresponding equivalent circuit used in the EIS analysis along with the (b) chemical structures of the DPTL and DPhPC molecules.

\subsection{EIS instrument and measurements}

EIS measurements were performed in the frequency region between $10^{4}$ and $10^{-2}$ $\mathrm{Hz}$ with a bias potential of $0.0 \mathrm{~V}$ vs $\mathrm{Ag} / \mathrm{AgCl}$. The details of the EIS measurements were described in Ref. [27]. The impedance spectra were fitted to an equivalent circuit proposed by Valincius et al. [28-32] as shown in Fig. 1(a). $R_{\text {sol }}$ is the resistance of the electrolyte, $R_{\mathrm{m}}$ and $C P E_{\mathrm{m}}$ represent the resistance and constant phase element of the membrane and $C P E_{\mathrm{sp}}$ represents the constant phase element of the spacer between the bilayer and gold (111) surface. The constant phase elements were used in the equivalent circuit to account for the heterogeneity of tBLMs. The impedance of the $C P E$ can be calculated as:

$$
Z_{C P E}=\frac{1}{Q(j \omega)^{\alpha}}
$$

where $Q$ is the constant phase element coefficient measured in $\mu \mathrm{F} \mathrm{cm}^{-2} \mathrm{~s}^{\alpha-1}$, and the 
exponent $\alpha$ is an empirical constant related to the frequency dispersion.

\subsection{PM-IRRAS experiments}

PM-IRRAS experiments were carried using Thermo Nicolet Nexus 870 spectrometer (Madison, WI), equipped with an external tabletop optical mount (TOM) box. The half-wave retardation of the PEM was set at $1600 \mathrm{~cm}^{-1}$ for the $\mathrm{C}=\mathrm{O}$ stretching region and $2900 \mathrm{~cm}^{-1}$ for the $\mathrm{C}-\mathrm{H}$ stretching region. The angle of incidence was placed at $60^{\circ}$ to obtain a large enhancement of the mean square electric field strength of the $p$-polarized radiation at gold surface. $\mathrm{D}_{2} \mathrm{O}$ electrolyte was employed to avoid the huge IR absorption of $\mathrm{H}_{2} \mathrm{O}$ around $1650 \mathrm{~cm}^{-1}$. At each electrode potential, $4000 \mathrm{IR}$ spectra were added and averaged using an instrumental resolution of $4 \mathrm{~cm}^{-1}$. The detailed information about the measurement and data processing in PM-IRRAS experiment was given in Ref. [33, 34].

PM-IRRAS measures the signal $\Delta S$, which is proportional to the absorbance, $A$, of the molecules adsorbed at the electrode surface, and is defined as:

$$
\Delta S=\frac{2\left(I_{s}-I_{p}\right)}{I_{s}+I_{p}}=2.3 A=2.3 \Gamma \varepsilon
$$

where $I_{s}$ and $I_{p}$ are the intensities of the $s$ - and $p$-polarized radiation, $\Gamma$ is the surface concentration of the adsorbed species and $\varepsilon$ is the decimal molar absorption coefficient of the adsorbed species. The normalized PM-IRRAS spectra $\left(\Delta S_{\text {nor }}\right)$ are defined as [27]:

$$
\Delta S_{\mathrm{nor}}=\frac{\Delta S}{<E_{p, \mathrm{z}=0}^{2}>/<E_{p 0}^{2}>}
$$

where $\left\langle E_{p 0}^{2}\right\rangle$ is the mean square electric field strength of the $p$-polarized incident beam and $\left\langle E_{p, z=0}^{2}\right\rangle$ is the mean square electric field strength of the $p$-polarized beam at the gold (111) electrode surface, which can be calculated by solving Fresnel equations for a model consisting of six stratified layers (Au/spacer/DPTL/Alm:DPhPC $\left.(x: 100-x) / \mathrm{D}_{2} \mathrm{O} / \mathrm{CaF}_{2}\right)$. The $\Delta S_{\text {nor }}$ spectra eliminate the influence of the optical setup on the PM-IRRAS spectra, such as the angle of incidence and separation distance between the $\mathrm{CaF}_{2}$ prism and the electrode. The corrected PM-IRRAS spectra plot only the IR 
contribution from adsorbed species at the electrode surface.

\subsection{Tilt angle calculations}

The PM-IRRAS spectra of the Alm:DPhPC(x:100-x)/DPTL tBLMs can be simulated by solving Fresnel equations for a model of six homogeneous, parallel phases (Au/spacer/DPTL/Alm:DPhPC/ $\left.\mathrm{D}_{2} \mathrm{O} / \mathrm{CaF}_{2}\right)$ using custom made software [35]. The optical constants of the Alm:DPhPC mixture were taken from Ref. [13]. The average tilt angles of the $3_{10}$-helix $\left(\gamma_{310}\right)$ and $\alpha$-helix $\left(\gamma_{\alpha}\right)$ in alamethicin molecules with respect to the surface normal can be calculated from the intensity of the corresponding IR absorption band in the measured and simulated PM-IRRAS spectra. The detail of the spectral simulation and tilt angle calculations are described in Ref. [13, 27].

\section{Results and Discussion}

\subsection{EIS results}

Fig. 2(a) plots the absolute values of the impedance and Fig. 2(b) shows the corresponding phase angles as a function of the frequency for the gold electrode covered by the Alm:DPhPC $(x: 100-x) / \mathrm{DPTL}$ bilayer with varying concentrations of alamethicin $(x \mathrm{~mol} \%)$ in $0.1 \mathrm{M} \mathrm{KClO}_{4}$ electrolyte. The impedance of the DPhPC/DPTL tBLM in the absence of alamethicin $(x=0)$ is very high indicating that DPhPC/DPTL forms a uniform bilayer at the gold (111) surface. After the incorporation of alamethicin into the tBLM, the total impedance of the tBLM decreases. At a fixed frequency of $5 \mathrm{~Hz}$, the change of the impedance as a function of the alamethicin concentration is shown in Fig. 3. The impedance decreases with increasing alamethicin concentration where it attains a minimum at $15 \mathrm{~mol} \%$ and then begins increasing at higher peptide content. The phase angle plots in Fig. 2(b) display a minimum in the low frequency region $(f<10 \mathrm{~Hz})$. Valincius et al. [31,32] have shown that the minimum in the phase angle plot corresponds to the formation of defects or pores in the tBLM. The logarithm of the minimum frequency $\left(f_{\min }\right)$ can be correlated with the defect (or pore) density. The top curve in Fig. 3, plots $\log f_{\min }$ as a function of alamethicin concentration 
in the bilayer. The curve displays a maximum at the concentration where the $|\mathrm{Z}|$ plot reaches a minimum. Therefore, the EIS data shows that alamethicin forms pores/defects in the tBLM where the highest pore/defect density is observed at 15 mol\% alamethicin. At higher peptide concentrations ( $\mathrm{x}>15 \mathrm{~mol} \%$ ), the total impedance increases suggesting that either the ability of alamethicin to form pores/defects decreases or that no further insertion can occur forming a mixed inserted and surface states.
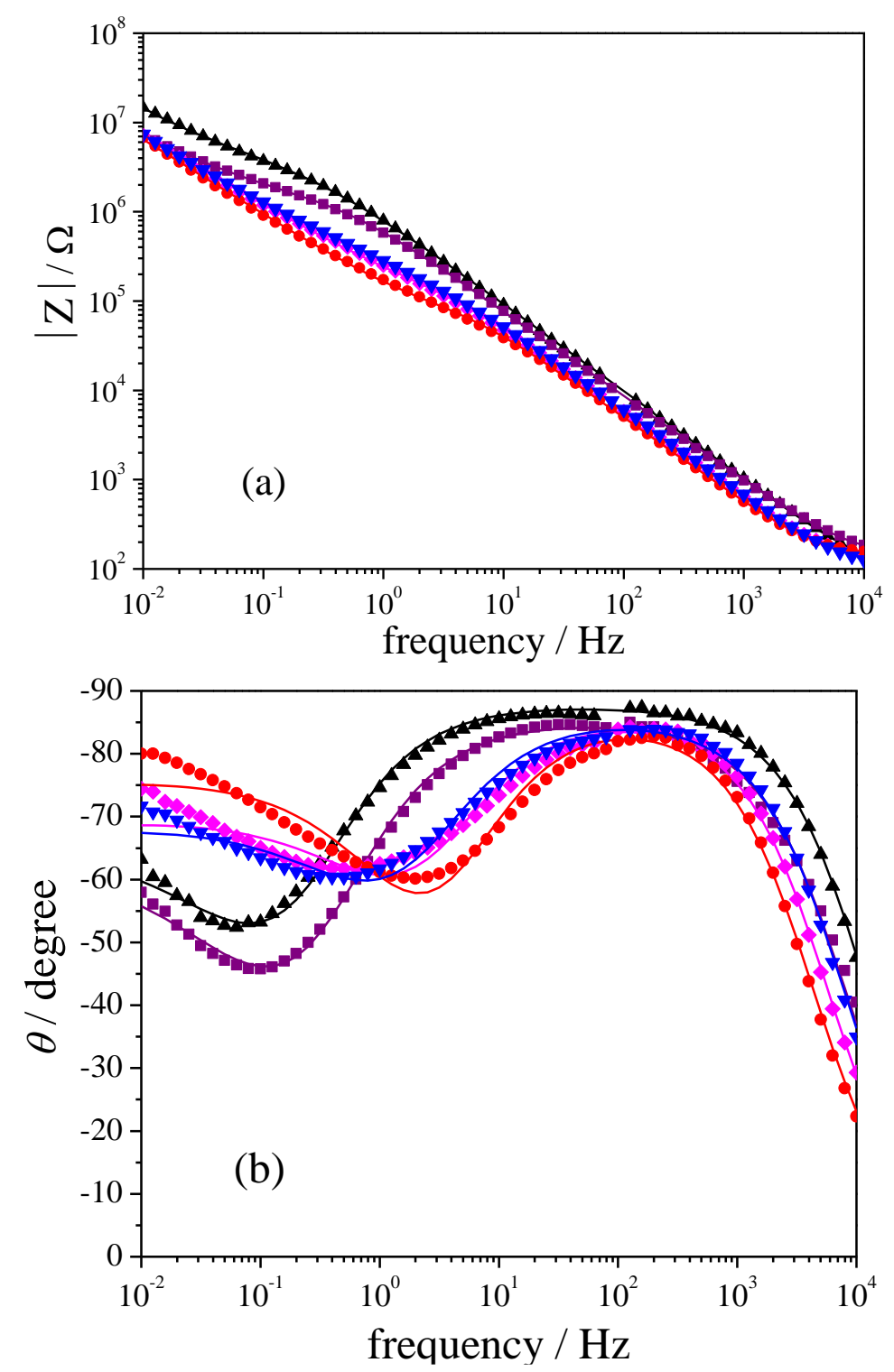

Fig. 2 EIS spectra of the Alm:DPhPC $(x: 100-x) / D P T L$ tBLMs showing the (a) magnitude of the total impedance and (b) phase angle of the different alamethicin concentrations $\left(x \mathrm{~mol} \%\right.$ ) in a $0.1 \mathrm{M} \mathrm{KClO}_{4}$ at $E=0.0 \mathrm{~V}$ vs $\mathrm{Ag} / \mathrm{AgCl}$. The alamethicin concentrations $(x)$ are represented by the following: 0 ( $\triangle$, black), 2.5 ( $\square$, purple), 10 
( $\diamond$, magenta), $15(\circ$, red) and $30(\nabla$, blue) where the solid lines show the resulting fit of the equivalent circuit presented in Fig. 1(a).

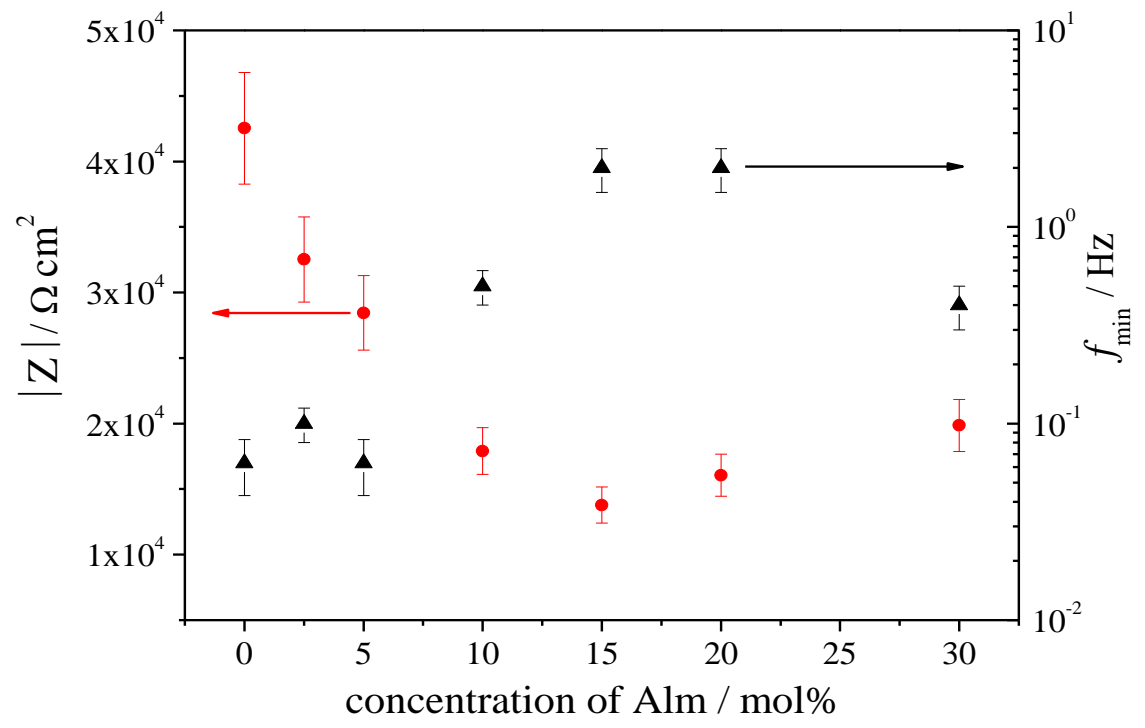

Fig. 3 Total impedance of the $\operatorname{Alm:DPhPC}(x: 100-x) / D P T L$ bilayer at a fixed frequency of $5 \mathrm{~Hz}$ (1(red circles; left axis) and the position of the frequency minimum in the phase angle (black triangles; right axis) as a function of the alamethicin concentration.

Further analysis of the EIS data requires fitting of the data to a model. The choice of a model is heuristic and based on the insight provided by the structure of the investigated bilayer [32]. A model represented by three parallel resistors and capacitors connected in series was used in our recent study of gramicidin incorporated into the DPhPC/DPTL bilayer [27]. In that study, gramicidin was present in both leaflets of the bilayer and each mesh of parallel resistors and capacitors could be assigned to the spacer, inner and outer leaflets of the tBLM. This equivalent circuit also fits the current data. However, alamethicin is spanning the membrane and it is physically incorrect to model the system as two individual leaflets. In addition, our previous model implicitly assumes that the inner side of the bilayer is equipotential. However, in the present model, the formation of membrane pores are heterogeneous and therefore, the ions that penetrate into the spacer layer will not uniformly distribute in the spacer, which implies that the inner leaflet cannot be equipotential [32]. Valincius et al. [28-30] proposed an equivalent circuit, shown in Fig. 1(a), to model EIS data for a tBLM. In this model, the 
equipotential plane is located inside the metal phase. The membrane resistance and capacitance are represented by $R_{\mathrm{m}}$ and $C P E_{\mathrm{m}}$ and the spacer by $C P E_{\mathrm{sp}}$. The Cole-Cole plots in Fig. 4 show that this model is in good agreement with present data for frequencies ranging between $10^{4}$ and $0.1 \mathrm{~Hz}$. The values of $R_{\mathrm{m}}, C P E_{\mathrm{m}}$ and $C P E_{\mathrm{sp}}$

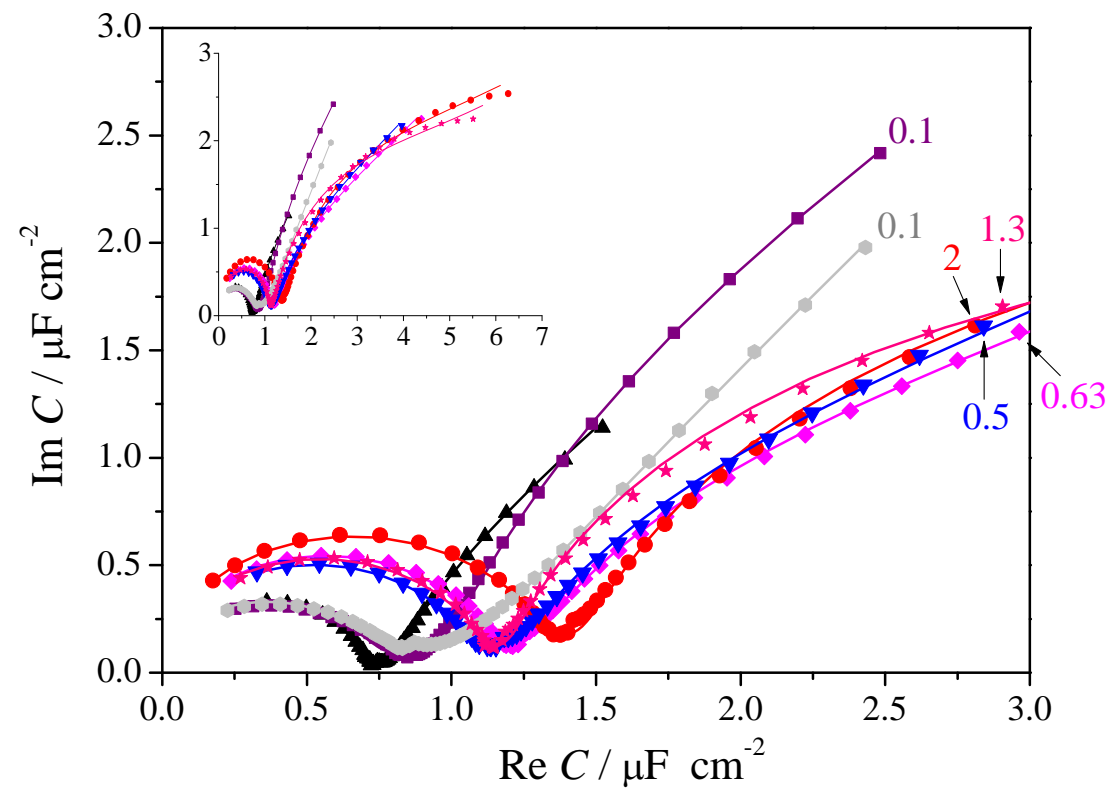

determined from the fit are listed in Table 1.

Fig. 4 Cole-Cole plots of the EIS data in the high frequency range for gold (111) electrode covered with $\operatorname{Alm}: \operatorname{DPhPC}(x: 100-x) / \mathrm{DPTL}$ bilayers for different alamethicin concentrations ( $x \mathrm{~mol} \%$ ) in $0.1 \mathrm{M} \mathrm{KClO}_{4}$ at $E=0.0 \mathrm{~V}$ vs $\mathrm{Ag} / \mathrm{AgCl}$, where the values of $x$ are: $0(\triangle$, black), 2.5 ( $\square$, purple), 5 ( $\square$, gray), $10(\diamond$, magenta), 15 ( $\circ$, red $), 20$ ( $\triangleleft$, pink) and $30(\nabla$, blue). Lines represent the fit to the equivalent circuit shown in Fig. 1(a). The numbers at each plot show the lowest frequency value corresponding to that curve. The inset in Fig. 4 shows the Cole-Cole plot in the whole frequency region from 0.1 to $10^{4}$ Hz.

The parameter $\alpha$ of $C P E_{\mathrm{m}}$ is close to unity and hence $C P E_{\mathrm{m}}$ values could be used as a good representation of the electrode capacitance. For the DPhPC/DPTL bilayer in the absence of alamethicin, the capacitance equal to $0.85 \pm 0.01 \mu \mathrm{F} \mathrm{cm}^{-2}$ was obtained, which is in a good agreement with the capacitance measured by Schiller et al. [4]. Upon incorporation of alamethicin, the capacitance increases indicating that the alamethicin alters the structure of the bilayer. The membrane resistance, $R_{\mathrm{m}}$, in the absence of alamethicin is quite low compared to traditional BLMs indicating that the tBLM 
consists of defects. The addition of alamethicin leads to a further decrease of $R_{\mathrm{m}}$ reaching a minimum value of $20.9 \mathrm{k} \Omega \mathrm{cm}^{2}$ at alamethicin content of $15 \mathrm{~mol} \%$. As the alamethicin content is further increased, the membrane resistance achieves higher values. The changes of $R_{\mathrm{m}}$ show a similar trend to that of the $|\mathrm{Z}|$ presented in Fig.3. This indicates that the incorporated alamethicin molecules allow for ion translocation across the membrane through the formation of defects or pores at sufficiently high peptide concentrations. The parameter $\alpha$ of the $C P E_{\mathrm{sp}}$ is close to 0.5 when the alamethicin concentration is less than $10 \%$. This value indicates that defect/pore density is considerably low and they are sufficiently isolated, so that ionic fluxes penetrating through individual pores do not overlap in the submembrane region [31]. At higher alamethicin content, $\alpha$ of the $C P E_{\mathrm{sp}}$ increases indicating an increase in the density of defects and/or ion conductive pores. The $C P E_{\mathrm{sp}}$ also increases indicating that water content in the region between the membrane and gold surface increases as well [31]. At frequencies below $0.1 \mathrm{~Hz}$, the fit of the EIS data to the equivalent circuit in Fig. 1(a) was poor since the ionic transport parallel to the electrode surface at these low frequencies contributes to the measured impedance. These deviations are most pronounced for $15 \mathrm{~mol} \%$ alamethicin content, which corresponds to the highest pore density (lowest membrane resistivity) and highest water content in the spacer (highest

Table 1 Numerical values for each of the elements in the equivalent circuit used to

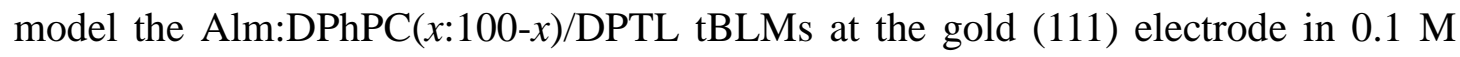
$\mathrm{KClO}_{4}$ at various alamethicin concentrations $(x \mathrm{~mol} \%)$ and $E=0.0 \mathrm{~V}$ vs $\mathrm{Ag} / \mathrm{AgCl}$.

\begin{tabular}{|c|c|c|c|c|c|c|c|}
\hline$x / \mathrm{mol} \%$ & 0 & $2.5 \%$ & $5.0 \%$ & $10 \%$ & $15 \%$ & $20 \%$ & $30 \%$ \\
\hline$Q$ of $C P E_{\mathrm{m}} /$ & $0.85 \pm$ & $1.14 \pm$ & $1.39 \pm$ & $1.54 \pm$ \\
$\mu \mathrm{F} \mathrm{cm}^{-2} \mathrm{~s}^{\alpha-1}$ & 0.01 & 0.02 & 0.02 & 0.01 & $\begin{array}{c}1.77 \pm \\
0.03\end{array}$ & $\begin{array}{c}1.43 \pm \\
0.04\end{array}$ & $\begin{array}{c}1.51 \pm \\
0.03\end{array}$ \\
\hline$\alpha$ of $C P E_{\mathrm{m}}$ & $\begin{array}{c}0.978 \pm \\
0.001\end{array}$ & $\begin{array}{c}0.951 \pm \\
0.002\end{array}$ & $\begin{array}{c}0.930 \pm \\
0.002\end{array}$ & $\begin{array}{c}0.967 \pm \\
0.002\end{array}$ & $\begin{array}{c}0.967 \pm \\
0.003\end{array}$ & $\begin{array}{c}0.971 \pm \\
0.003\end{array}$ & $\begin{array}{c}0.961 \pm \\
0.002\end{array}$ \\
\hline$R_{\mathrm{m}} / \mathrm{k} \Omega \mathrm{cm}^{2}$ & $491 \pm$ & $273 \pm$ & $232 \pm$ & $31.5 \pm$ & $20.9 \pm$ & $22.9 \pm$ & $45.5 \pm$ \\
26 & 26 & 24 & 2.0 & 1.0 & 1.0 & 2.6 \\
\hline$Q$ of CPE $\mathrm{sp} /$ & $1.48 \pm$ & $3.26 \pm$ & $2.13 \pm$ & $4.15 \pm$ \\
$\mu \mathrm{F} \mathrm{cm}^{-2} \mathrm{~s}^{\alpha-1}$ & 0.06 & 0.26 & 0.16 & 0.04 & $\begin{array}{c}5.68 \pm \\
0.08\end{array}$ & $\begin{array}{c}5.41 \pm \\
0.08\end{array}$ & $\begin{array}{c}3.86 \pm \\
0.04\end{array}$ \\
\hline
\end{tabular}




\begin{tabular}{|c|c|c|c|c|c|c|c|}
\hline \multirow{2}{*}{$\alpha$ of $C P E_{\mathrm{sp}}$} & $0.514 \pm$ & $0.537 \pm$ & $0.441 \pm$ & $0.652 \pm$ & $0.741 \pm$ & $0.748 \pm$ & $0.641 \pm$ \\
& 0.012 & 0.017 & 0.021 & 0.008 & 0.008 & 0.008 & 0.009 \\
\hline
\end{tabular}

value of $\left.C P E_{\mathrm{sp}}\right)$ as expected. This suggests that a more complex model is required to account for the specific conductivity and thickness of the spacer region as well as the density and distribution of the alamethicin pores [32]. Since the specific conductivity of the spacer region, pore density and pore distribution are not known, calculations in this frequency domain have not been performed.

\subsection{PM-IRRAS result}

The EIS results demonstrated that alamethicin incorporated into the DPhPC/DPTL bilayer forms defects or pores where the highest number of conductive channels was observed at concentrations between 10 and $20 \mathrm{~mol} \%$. To determine whether the increase in conductivity was related to formation of defects or pores, PM-IRRAS was utilized to obtain the molecular level information about the conformation and orientation of alamethicin in the tBLMs to complement our EIS results.

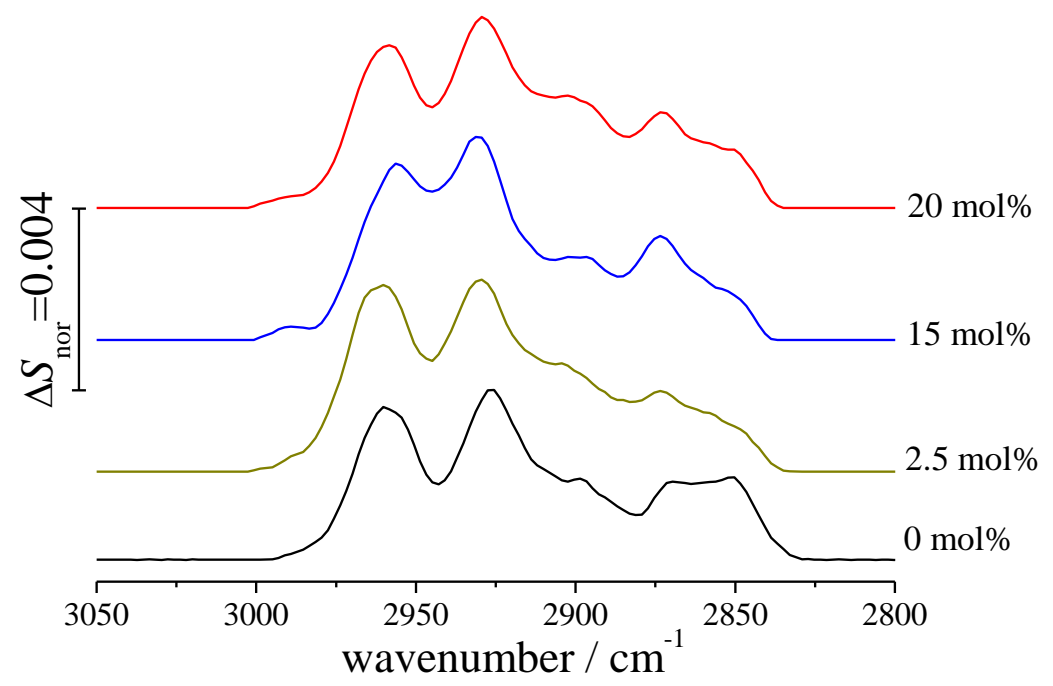

Fig. 5 Normalized PM-IRRAS spectra of the diphytanyl chains in the Alm:DPhPC $(x: 100-x) / \mathrm{DPTL}$ bilayers tethered to the gold (111) electrode at $E=0.0 \mathrm{~V}$ vs $\mathrm{Ag} / \mathrm{AgCl}$ in in $0.1 \mathrm{M} \mathrm{KClO}_{4} / \mathrm{D}_{2} \mathrm{O}$ for varying concentrations of alamethicin (denoted by the $\mathrm{mol} \%$ ) at.

Fig. 5 displays the normalized PM-IRRAS spectra $\left(\Delta S_{\text {nor }}\right)$ of diphytanyl chains 
Alm:DPhPC $(x: 100-x) / \mathrm{DPTL}$ tethered bilayers at the gold (111) electrode surface at selected peptide concentrations. The $\Delta S_{\text {nor }}$ spectra eliminate the influence of the electric field enhancement from the optical setup from the experimental PM-IRRAS spectra to allow quantitative comparisons to be made between $\operatorname{Alm:DPhPC}(x: 100-x) / \mathrm{DPTL}$ spectra collected under different experimental conditions. The intensity of the symmetric and asymmetric $\mathrm{CH}_{2}$ stretching bands can be used to determine the average tilt angle of trans fragments of the hydrophobic tails with respect to the surface normal, using the procedure described in [13]. The tilt angles of trans fragments can be used to approximate the tilt angle of the hydrocarbon chains, which are listed in Table 2. The IR measurements suggest that the presence of alamethicin in the DPhPC/DPTL bilayer results an overall increase in the average tilt angle of the bilayer at all selected potentials. This behavior indicates that alamethicin causes a thinning of the bilayer and is supported by the previous EIS measurements, which show an increase in the $C P E_{\mathrm{m}}$ upon insertion of alamethicin since capacitance is inversely proportional to the bilayer thickness (i.e. $C=\varepsilon / d$ where $\varepsilon$ is the permittivity and $d$ is the bilayer thickness). Indeed, the largest tilt angle of the hydrocarbon chains is observed for alamethicin content of 15 mol\%, which also corresponds to the highest value $C P E_{\mathrm{m}}$ value in Table 1.

Table 2 Tilt angles of the diphytanyl chains of the Alm:DPhPC $(x: 100-x) / \mathrm{DPTL}$ tethered bilayers on the gold (111) electrode surface for selected peptide concentrations. .

\begin{tabular}{|c|c|}
\hline $\begin{array}{l}\text { Concentration of } \\
\text { Alm / mol\% } \%\end{array}$ & $\begin{array}{l}\text { Tilt angle of the } \\
\text { diphytanyl chain }\end{array}$ \\
\hline 0 & $31 \pm 4^{\circ}$ \\
\hline 2.5 & $35 \pm 4^{\circ}$ \\
\hline 15 & $38 \pm 4^{\circ}$ \\
\hline 20 & $36 \pm 4^{\circ}$ \\
\hline
\end{tabular}

Additional information about the membrane properties is provided by the analysis of the amide I band of the peptide. Fig. 6 displays the normalized PM-IRRAS spectra $\left(\Delta S_{\text {nor }}\right)$ of the amide $\mathrm{I}$ and $\mathrm{C}=\mathrm{O}$ vibrational bands for variable alamethicin concentrations $(x \mathrm{~mol} \%)$ in the tBLM. The spectrum of the pure DPhPC/DPTL bilayer 
( $x=0$ ) displays a single IR band between 1700 and $1750 \mathrm{~cm}^{-1}$ corresponding to the carbonyl stretching in the lipid molecules. After the incorporation of alamethicin into the tBLMs, a broad amide I band between 1600 and $1700 \mathrm{~cm}^{-1}$ appears. The deconvolution of amide I band is presented in Fig. 7 and shows that the spectral envelope is composed of five individual sub-bands [13, 36-38]. The two strong bands appearing at 1635 and $1659 \mathrm{~cm}^{-1}$ correspond to the 310 -helix and $\alpha$-helix, respectively. The band at $1620 \mathrm{~cm}^{-1}$ is assigned to the $\beta$-sheet structure, and two bands at 1647 and $1674 \mathrm{~cm}^{-1}$ are related to the type III $\beta$-turn structure associated with single turn of the 310 -helix [37]. The bands corresponding to the 310 -helix and $\alpha$-helix are much stronger than the other three bands. This is consistent with XRD data, which shows that the secondary structure of alamethicin is predominantly helical in nature [9]. 


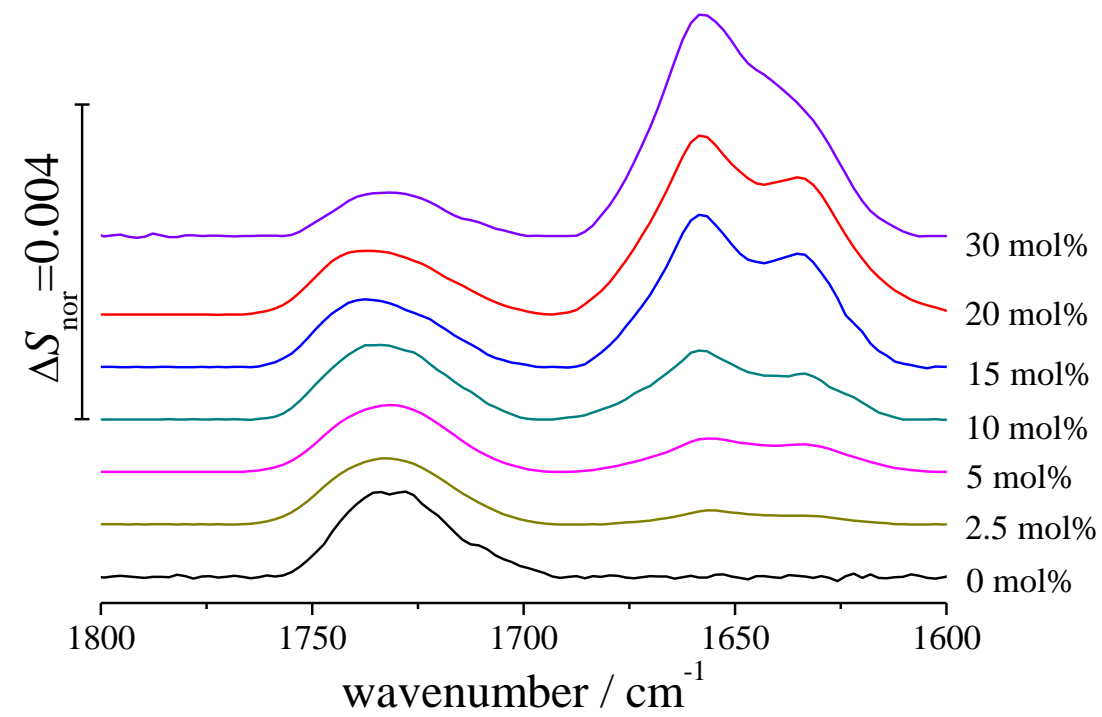

Fig. 6 Normalized amide I and carbonyl spectral bands from the PM-IRRAS spectra of the Alm:DPhPC $(x: 100-x) / \mathrm{DPTL}$ tBLMs at different concentration of alamethicin (denoted by the $\mathrm{mol} \%$ ) in $0.1 \mathrm{M} \mathrm{KClO}_{4} / \mathrm{D}_{2} \mathrm{O}$ at $E=0.0 \mathrm{~V}$ vs $\mathrm{Ag} / \mathrm{AgCl}$.

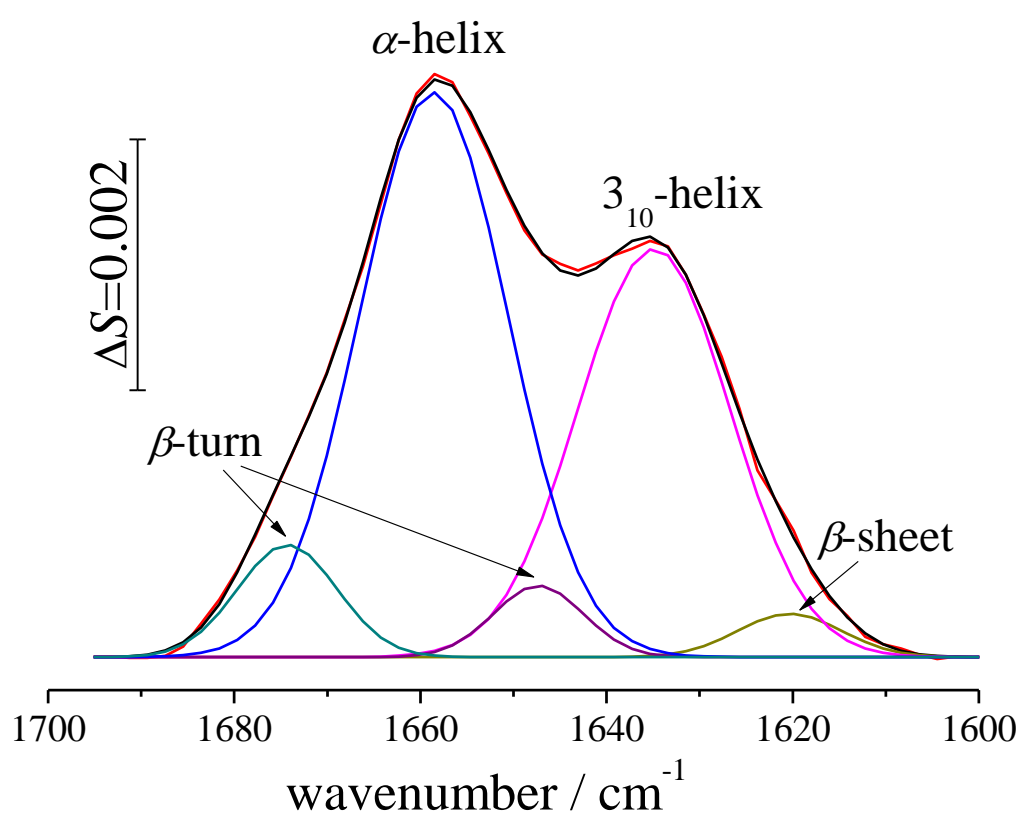

Fig. 7 Deconvolution of the amide I band of the Alm:DPhPC(15:85)/DPTL tethered bilayer at in $0.1 \mathrm{M} \mathrm{KClO}_{4} / \mathrm{D}_{2} \mathrm{O}$ at $E=0.0 \mathrm{~V}$ vs $\mathrm{Ag} / \mathrm{AgCl}$. 
The integrated intensity of the two prominent amide bands at 1635 and $1659 \mathrm{~cm}^{-1}$ are used to calculate the tilt angles of the 310 -helix $\left(\gamma_{310}\right)$ and $\alpha$-helix $\left(\gamma_{\alpha}\right)$, using the procedure described in Ref. [13]. Fig. 8 displays the tilt angles of the $\gamma_{310}$ and $\gamma_{\alpha}$ as a function of alamethicin concentration in the tethered bilayers. At low alamethicin concentrations, i.e. $2.5 \mathrm{~mol} \%$, the values of $\gamma_{310}$ and $\gamma_{\alpha}$ are $82 \pm 3^{\circ}$ and $64 \pm 3^{\circ}$, respectively. These high values indicate that alamethicin adopts surface state with helices assuming small angle with respect to the bilayer surface. When alamethicin concentration increases, the values of $\gamma_{310}$ and $\gamma_{\alpha}$ decrease and at a peptide concentration of $15 \mathrm{~mol} \%$ reach a minimum of $59 \pm 3^{\circ}$ and $31 \pm 4^{\circ}$, respectively. The low tilt angle values with respect to surface normal indicate that the alamethicin peptides are inserting into the bilayer. At all concentrations, the difference between the tilt angle of the 310 -helix and $\alpha$-helix $\left(\gamma_{310-} \gamma_{\alpha}\right)$ ranges between $20 \pm 5^{\circ}$ to $30 \pm 5^{\circ}$. The estimated angle of the proline kink linking the 310 -helix to the $\alpha$-helix lies between $15^{\circ}$ and $25^{\circ}$, which is in good agreement with our results $[9,39]$. To correlate IR and EIS data, the values of membrane resistance, reported in Table 1, are also plotted in Fig. 8. The minimum in the $R_{m}$ plot occurs also at alamethicin concentration of $15 \mathrm{~mol} \%$. The EIS results show an excellent correlation with the PM IRRAS measurements. This correlation indicates that increased membrane conductivity (decrease of $R_{\mathrm{m}}$ ) is caused by the formation of alamethicin pores rather than an increased presence of membrane defects. 


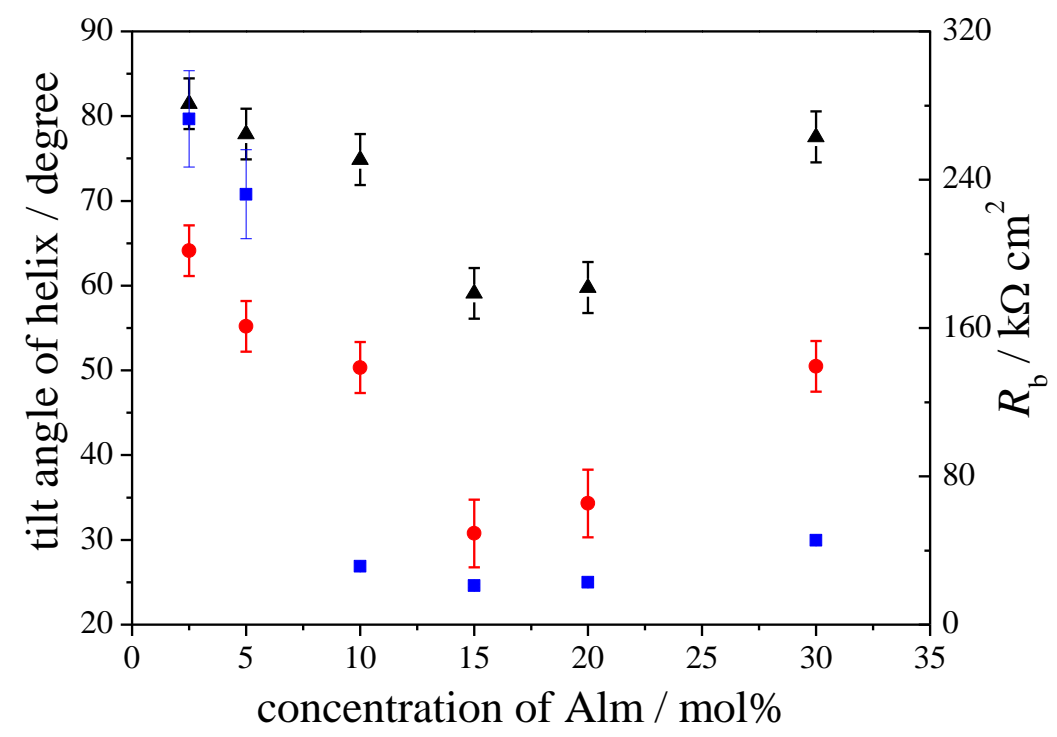

Figure 8 The tilt angles of the 310 -helix (black triangles) and $\alpha$-helix structure (red circles) are shown on the left axis and membrane resistance, $R_{\mathrm{m}}$, values (blue rectangles) are displayed on the right axis for $\operatorname{Alm}: \operatorname{DPhPC}(x: 100-x) / \mathrm{DPTL}$ tBLMs as a function of the alamethicin concentration.

The PM-IRRAS results are in good agreement with the orientation data obtained by the Huang research group using oriented circular dichroism (OCD) to study alamethicin incorporated into DPhPC bilayers. [40]. Their results demonstrate that alamethicin adopts a surface state at low concentrations and inserts into the DPhPC bilayer at high concentrations. The Chen laboratory employed sum frequency generation spectroscopy to study the insertion of alamethicin into POPC and d-DMPC bilayers. Their results also confirmed that alamethicin forms a surface state at low concentrations and a bilayer inserted state at high concentrations [22, 23].

Recently, ion channel properties of alamethicin were investigated in the DPhPC bilayer supported at a bare gold (111) surface (sBLM) [13]. These results demonstrated that alamethicin insertion into the membrane strongly depends on the electrode potential. The alamethicin molecules assume a surface orientation at positive transmembrane potentials and insert into the bilayer and aggregate to form ion conductive pores at negative transmembrane potentials. To investigate the effect of 
electrode potential on the insertion of alamethicin into the membrane, PM-IRRAS spectra, plotted in Fig. 9, were collected as a function of the electrode potential for the Alm:DPhPC(15:85)/DPTL tethered bilayer.

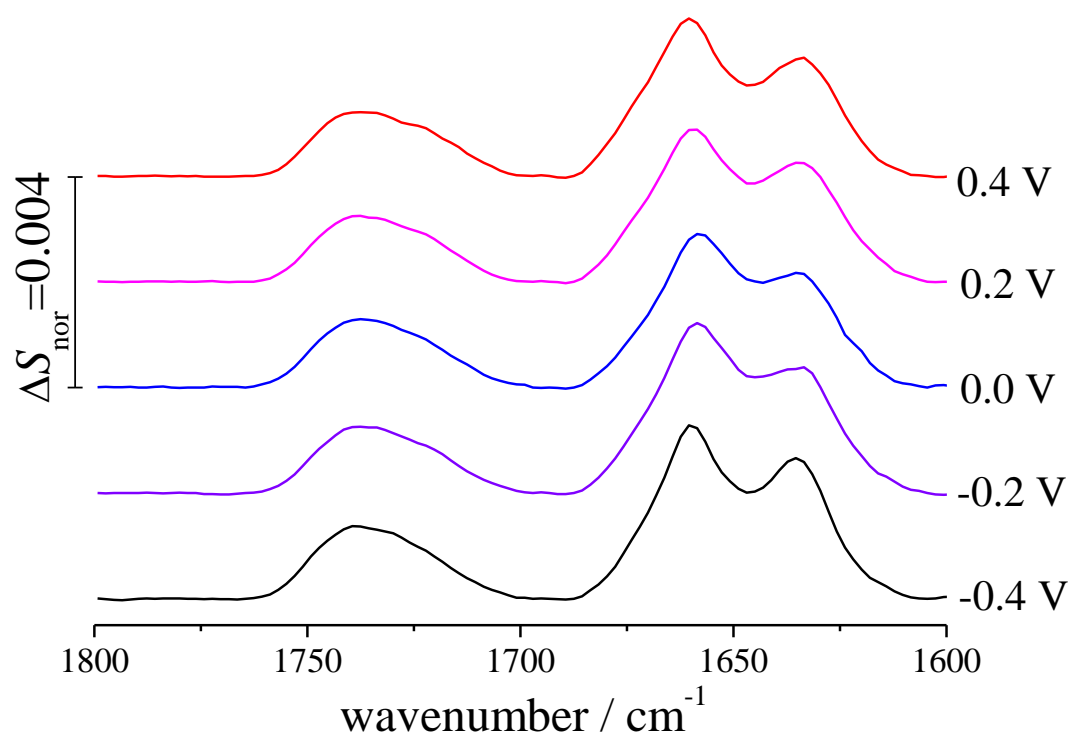

Figure 9 Normalized PM-IRRAS spectra of the Alm:DPhPC(15:85)/DPTL tBLM at different electrode potentials measured versus $\mathrm{Ag} / \mathrm{AgCl}$.

The intensity of the amide I band displays a small change with the electrode potential in tBLM, in strong contrast to the behavior of this peptide in the sBLM. This behavior indicates that either the mobility of alamethicin is much lower in the tBLM, or that the large pore density allows ions to penetrate into the spacer between the membrane and the metal surface. In that case, a significant portion of the potential drop between the metal and electrolyte takes place within the spacer rather than across the membrane. Incidentally, the antibiotic properties of the peptide are related to its ability to depolarize the transmembrane potential. The absence of a potential dependence on the insertion of the into the bilayer was also observed for gramicidin $\mathrm{A}$ in the DPhPC/DPTL tethered bilayer [27]. However, gramicidin displayed a much different concentration dependence where it behaved as the ion channel at concentrations below $15 \mathrm{~mol} \%$, and adopted a non-conductive state at the concentration of $20 \mathrm{~mol} \%$. Furthermore, the ion transport properties of alamethicin and gramicidin are different. Gramicidin forms ion channel allowing selective transport of monovalent cations when 
the two molecules present in the each separate leaflet form a dimer, which means that they act as single channels dispersed in the lipid matrix [41-43]. In contrast, alamethicin molecules aggregate to form ion pores that span the entire bilayer [44-46]. Consequently, the concentration dependence of the two peptides is different when they are inserted into the bilayer.

\section{Conclusion}

EIS and PM-IRRAS techniques were employed to investigate the ion pore properties of alamethicin incorporated into the DPhPC/DPTL tethered bilayer as a function of the alamethicin concentration. The results indicate that at low concentration, alamethicin adopts a surface state with high tilt angles of the 310 -helix and $\alpha$-helix. The tilt angle decreases with increasing peptide concentration achieving a minimum value at an alamethicin content of $15 \mathrm{~mol} \%$ where the peptide is inserted into the membrane. At higher alamethicin concentrations, the tilt angle increases to larger values indicating a poorer insertion of peptides into the membrane or formation of a mixture of inserted and surface state. The changes in the peptide orientation correlate very well with the membrane conductivity data. The helices adopt a large tilt angles when the membrane conductivity is low (i.e. high membrane resistivity) and low tilt angles when the conductivity is high (i.e. low membrane resistivity). The combination of PM-IRRAS and EIS measurements provides direct evidence that alamethicin forms ion conductive pores in the tethered bilayer. The interpretation of the EIS measurements is highly model dependent and requires verification from additional analytical methodologies. The good correlation between IR spectroscopic and electrochemical data validates the model equivalent circuit used to analyze the EIS measurements. These findings demonstrate the power of a two-pronged approach (i.e. complementary experiments) to study membrane-active peptides and proteins in a quasi-natural environment. In striking contrast to the behavior of alamethicin in the freely suspended gold supported bilayer [13], in so-called black lipid membranes [11] and in mercury supported tBLM [47], the tilt angle of the alamethicin molecules inserted into the tethered bilayer (15 
mol\% Alm) do not exhibit a dependence on the electrode potential. This behavior indicates that either the mobility of molecules in the tethered bilayer is poor, or that a sufficient number of ions penetrate into the spacer between the membrane and the metal causing the primary potential drop to occur between the metal and thin layer of electrolyte within the spacer region.

\section{Acknowledgements}

This work was supported by a Discovery grant from Natural Sciences and Engineering Research Council of Canada (NSERC). JL acknowledges helpful discussion of EIS data with Prof Manuela Ruda and Prof Francesco Prieto from the University of Sevilla, Spain.

\section{References}

[1] J. Zhao, X. Zhao, Z. Jiang, Z. Li, X. Fan, J. Zhu, H. Wu, Y. Su, D. Yang, F. Pan, Biomimetic and bioinspired membranes: preparation and application, Prog. Polym. Sci. 39(9) (2014) 1668-1720.

[2] Y.-x. Shen, P.O. Saboe, I.T. Sines, M. Erbakan, M. Kumar, Biomimetic membranes: a review, J. Membr. Sci. 454 (2014) 359-381.

[3] S. Rebaud, O. Maniti, A.P. Girard-Egrot, Tethered bilayer lipid membranes (tBLMs): interest and applications for biological membrane investigations, Biochimie 107 (2014) 135-142.

[4] S.M. Schiller, R. Naumann, K. Lovejoy, H. Kunz, W. Knoll, Archaea analogue thiolipids for tethered bilayer lipid membranes on ultrasmooth gold surfaces, Angew. Chem. Int. Ed. 42(2) (2003) 208-211.

[5] R.L.C. Naumann, C. Nowak, W. Knoll, Proteins in biomimetic membranes: promises and facts, Soft Matter 7(20) (2011) 9535-9548.

[6] J.A. Jackman, W. Knoll, N.-J. Cho, Biotechnology applications of tethered lipid bilayer membranes, Materials 5(12) (2012) 2637-2657.

[7] W. Knoll, I. Köper, R. Naumann, E.-K. Sinner, Tethered bimolecular lipid membranes-A novel model membrane platform, Electrochim. Acta 53(23) (2008) 6680-6689.

[8] C. Meyer, F. Reusser, A polypeptide antibacterial agent isolated fromTrichoderma viride, Cell. Mol. Life Sci. 23(2) (1967) 85-86.

[9] R.O. Fox, F.M. Richards, A voltage-gated ion channel model inferred from the crystal structure of alamethicin at 1.5- $\AA$ resolution, Nature 300(5890) (1982) 325-330.

[10] P. Mueller, D.O. Rudin, Action potentials induced in biomolecular lipid membranes, Nature 217(5130) (1968) 713-719.

[11] R. Guidelli, L. Becucci, Mechanism of voltage-gated channel formation in lipid membranes, Biochim. Biophys. Acta 1858(4) (2016) 748-755. 
[12] H. Duclohier, H. Wroblewski, Voltage-dependent pore formation and antimicrobial activity by alamethicin and analogues, J. Membr. Biol. 184(1) (2001) 1-12.

[13] Z. Su, M. Shodiev, J. Jay Leitch, F. Abbasi, J. Lipkowski, In situ electrochemical and PM-IRRAS studies of alamethicin ion channel formation in model phospholipid bilayers, J. Electroanal. Chem. https://doi.org/10.1016/j.jelechem.2017.10.042.

[14] G. Baumann, P. Mueller, A molecular model of membrane excitability, J. Cell. Biochem. 2(5 - 6) (1974) 538-557.

[15] L. Bruner, A three state model for alamethicin conductance in bilayer membranes, J. Theor. Biol. 117(2) (1985) 265-276.

[16] H.W. Huang, Y. Wu, Lipid-alamethicin interactions influence alamethicin orientation, Biophys. J. 60(5) (1991) 1079-1087.

[17] K. He, S.J. Ludtke, W.T. Heller, H.W. Huang, Mechanism of alamethicin insertion into lipid bilayers, Biophys. J. 71(5) (1996) 2669-2679.

[18] T. Salditt, C. Li, A. Spaar, Structure of antimicrobial peptides and lipid membranes probed by interface-sensitive X-ray scattering, Biochim. Biophys. Acta 1758(9) (2006) 1483-1498.

[19] C. Li, T. Salditt, Structure of magainin and alamethicin in model membranes studied by X-ray reflectivity, Biophys. J. 91(9) (2006) 3285-3300.

[20] D. Constantin, G. Brotons, A. Jarre, C. Li, T. Salditt, Interaction of alamethicin pores in DMPC bilayers, Biophys. J. 92(11) (2007) 3978-3987.

[21] J. Pan, S. Tristram-Nagle, J.F. Nagle, Alamethicin aggregation in lipid membranes, J. Membr. Biol. 231(1) (2009) 11.

[22] P. Yang, F.-G. Wu, Z. Chen, Lipid fluid-gel phase transition induced alamethicin orientational change probed by sum frequency generation vibrational spectroscopy, J. Phys. Chem. C 117(33) (2013) 17039-17049.

[23] P. Yang, F.-G. Wu, Z. Chen, Dependence of alamethicin membrane orientation on the solution concentration, J. Phys. Chem. C 117(7) (2013) 3358-3365.

[24] J. Kunze, J. Leitch, A.L. Schwan, R.J. Faragher, R. Naumann, S. Schiller, W. Knoll, J.R. Dutcher, J. Lipkowski, New method to measure packing densities of self-assembled thiolipid monolayers, Langmuir 22(12) (2006) 5509-5519.

[25] J. Richer, J. Lipkowski, Measurement of physical adsorption of neutral organic species at solid electrodes, J. Electrochem. Soc. 133(1) (1986) 121-128.

[26] Y. Barenholz, D. Gibbes, B. Litman, J. Goll, T. Thompson, F. Carlson, A simple method for the preparation of homogeneous phospholipid vesicles, Biochemistry 16(12) (1977) 2806-2810.

[27] Z. Su, J.J. Leitch, R.J. Faragher, A.L. Schwan, J. Lipkowski, Gramicidin A ion channel formation in model phospholipid bilayers tethered to gold (111) electrode surfaces, Electrochim. Acta 243 (2017) 364-373.

[28] G. Valincius, D.J. McGillivray, W. Febo-Ayala, D.J. Vanderah, J.J. Kasianowicz, M. Lösche, Enzyme activity to augment the characterization of tethered bilayer membranes, J. Phys. Chem. B $110(21)$ (2006) 10213-10216.

[29] D.J. McGillivray, G. Valincius, D.J. Vanderah, W. Febo-Ayala, J.T. Woodward, F. Heinrich, J.J. Kasianowicz, M. Löscheb, Molecular-scale structural and functional characterization of sparsely tethered bilayer lipid membranes, Biointerphases 2(1) (2007) 21-33.

[30] D.J. McGillivray, G. Valincius, F. Heinrich, J.W. Robertson, D.J. Vanderah, W. Febo-Ayala, I. Ignatjev, M. Lösche, J.J. Kasianowicz, Structure of functional Staphylococcus aureus $\alpha$-hemolysin 
channels in tethered bilayer lipid membranes, Biophys. J. 96(4) (2009) 1547-1553.

[31] G. Valincius, T. Meskauskas, F. Ivanauskas, Electrochemical impedance spectroscopy of tethered bilayer membranes, Langmuir 28(1) (2012) 977-990.

[32] G. Valincius, M. Mickevicius, Tethered phospholipid bilayer membranes: an interpretation of the electrochemical impedance response, in: A. Iglic, C. Kulkarni, M. Rappolt (Eds.), Advances in Planar Lipid Bilayers and Liposomes, Academic Press, Burlington, 2015, pp. 27-61.

[33] V. Zamlynny, J. Lipkowski, Quantitative SNIFTIRS and PM IRRAS of organic molecules at electrode surfaces, in: R. Alkire, D.M. Kolb, L. J. (Eds.), Advances in Electrochemical Science and Engineering: Diffraction and Spectroscopic Methods in Electrochemistry, Wiley-VCH, Weinheim, 2006, pp. 315-376.

[34] A.H. Kycia, Z. Su, C.L. Brosseau, J. Lipkowski, In Situ PM-IRRAS Studies of Biomimetic Membranes Supported at Gold Electrode Surfaces, in: A. Wieckowski, C. Korzeniewski, B. Braunschweig (Eds.) Vibrational Spectroscopy at Electrified Interfaces, Wiley-VCH, Weinheim, 2013, pp. 345-417.

[35] V. Zamlynny, PhD thesis, University of Guelph (Canada), 2002.

[36] P.I. Haris, D. Chapman, Fourier transform infrared spectra of the polypeptide alamethicin and a possible structural similarity with bacteriorhodopsin, Biochim. Biophys. Acta 943(2) (1988) 375-380.

[37] D. Kennedy, M. Crisma, C. Toniolo, D. Chapman, Studies of peptides forming 310-and. alpha.-helixes and. beta.-bend ribbon structures in organic solution and in model biomembranes by Fourier transform infrared spectroscopy, Biochemistry 30(26) (1991) 6541-6548.

[38] S. Ye, K.T. Nguyen, Z. Chen, Interactions of alamethicin with model cell membranes investigated using sum frequency generation vibrational spectroscopy in real time in situ, J. Phys. Chem. B 114(9) (2010) 3334-3340.

[39] T. Nagao, D. Mishima, N. Javkhlantugs, J. Wang, D. Ishioka, K. Yokota, K. Norisada, I. Kawamura, K. Ueda, A. Naito, Structure and orientation of antibiotic peptide alamethicin in phospholipid bilayers as revealed by chemical shift oscillation analysis of solid state nuclear magnetic resonance and molecular dynamics simulation, Biochim. Biophys. Acta 1848(11) (2015) 2789-2798.

[40] F.-Y. Chen, M.-T. Lee, H.W. Huang, Sigmoidal concentration dependence of antimicrobial peptide activities: a case study on alamethicin, Biophys. J. 82(2) (2002) 908-914.

[41] E. Bamberg, K. Noda, P. Läuger, Single-channel parameters of gramicidin A, B, and C, Biochim. Biophys. Acta 419(2) (1976) 223-228.

[42] S. Weinstein, B. Wallace, E. Blout, J. Morrow, W. Veatch, Conformation of gramicidin A channel in phospholipid vesicles: a 13C and 19F nuclear magnetic resonance study, Proc. Nat.l Acad. Sci. U.S.A. 76(9) (1979) 4230-4234.

[43] S. Sek, T. Laredo, J.R. Dutcher, J. Lipkowski, Molecular resolution imaging of an antibiotic peptide in a lipid matrix, J. Am. Chem. Soc. 131(18) (2009) 6439-6444.

[44] K. He, S.J. Ludtke, D.L. Worcester, H.W. Huang, Neutron scattering in the plane of membranes: structure of alamethicin pores, Biophys. J. 70(6) (1996) 2659-2666.

[45] P. Pieta, J. Mirza, J. Lipkowski, Direct visualization of the alamethicin pore formed in a planar phospholipid matrix, Proc. Nat.1 Acad. Sci. U.S.A. 109(52) (2012) 21223-21227.

[46] S. Futaki, D. Noshiro, T. Kiwada, K. Asami, Extramembrane control of ion channel peptide assemblies, using alamethicin as an example, Acc. Chem. Res. 46(12) (2013) 2924-2933.

[47] L. Becucci, M. Papini, D. Mullen, A. Scaloni, G. Veglia, R. Guidelli, Probing membrane permeabilization by the antimicrobial peptide distinctin in mercury-supported biomimetic membranes, 
Biochim. Biophys. Acta 1808(11) (2011) 2745-2752. 